\title{
FIXED POINTS OF SEVERAL CLASSES OF NONLINEAR MAPPINGS IN BANACH SPACE
}

\author{
PETER K. F. KUHFITTIG
}

\begin{abstract}
In the first part of the paper conditions for the existence of ordinary and higher order fixed points of individual and commutative families of nonlinear operators are obtained.

The second part deals with the existence of fixed points of an operator $T: C \rightarrow X$ whose graph is closed in the Cartesian product topology induced by the strong topology in $C$ and the weak topology in $X$.

The convergence to fixed points of sequences of successive approximations is considered in both parts.
\end{abstract}

1. Introduction. The classical fixed-point theorem of Schauder states that if $T: C \rightarrow C$ is a continuous mapping of a closed convex subset of a Banach space $X$ into itself and if $T(C)$ is contained in a compact subset of $C$, then there exists an $x \in C$ such that $T x=x$. This result has been extended by Kirk [5] and others.

The purpose of this paper is to obtain additional conditions for the existence of fixed points, including fixed points of higher order. Common fixed points of families of operators and the convergence of sequences of successive approximations are also considered.

The following definitions will be required:

Definition 1. Let $X$ be a Banach space and $K$ a set in $X$. A mapping $T: K \rightarrow X$ is called nonexpansive if $\|T x-T y\| \leqq\|x-y\|$ for all $x$ and $y$ in $K$.

Definition 2. A Banach space is called strictly convex if for any pair of elements $x, y \in X$ from $\|x+y\|=\|x\|+\|y\|$ it follows that $x=\lambda y$, $\lambda>0$ (or, in the trivial case, $y=0$ ).

DEFINITION 3. If $T$ is a mapping on a Banach space, a point satisfying the condition $T^{n} x=x$ for some positive integer $n$ is called a fixed point of order $n$.

Definition 4. A family $\left\{T_{\lambda}\right\}_{\lambda \in \Lambda}$ of mappings of a set $K$ into itself is called commutative if $T_{\lambda} T_{\mu}=T_{\mu} T_{\lambda}$ for all $\lambda, \mu$ in $\Lambda$.

Received by the editors September 25, 1972 and, in revised form, October 1, 1973. AMS (MOS) subject classifications (1970). Primary 47H10; Secondary $45 \mathrm{G} 99$.

Key words and phrases. Nonexpansive mappings, fixed points.

(c) American Mathematical Society 1974 
2. Fixed points of order $n$. Let $T$ be nonexpansive. Since

$$
\left\|T^{n} x-T^{n} y\right\|=\left\|T\left(T^{n-1} x\right)-T\left(T^{n-1} y\right)\right\| \leqq\left\|T^{n-1} x-T^{n-1} y\right\|,
$$

it follows that $\left\|T^{n} x-T^{n} y\right\| \leqq i x-y \|$. As a consequence, higher order fixed points possess many properties of ordinary fixed points. For example, following the procedure for ordinary fixed points, one can show:

Proposition 1. If $C$ is a closed convex subset of a strictly convex Banach space $X$, then for every nonexpansive mapping $T_{\lambda}: C \rightarrow X$, the set $F_{\lambda}^{n}=\left\{x \in C: T_{\lambda}^{n} x=x\right\}$ is convex and closed.

Proof. If $x, y \in F_{\lambda}^{n}$, then for $z$ on the segment joining $x$ and $y$, we have

$$
\begin{aligned}
\|x-y\| & \leqq\left\|x-T_{\lambda}^{n} z\right\|+\left\|T_{\lambda}^{n} z-y\right\| \\
& =\left\|T_{\lambda}^{n} x-T_{\lambda}^{n} z\right\|+\left\|T_{\lambda}^{n} z-T_{\lambda}^{n} y\right\| \\
& \leqq\|x-z\|+\|z-y\|=\|x-y\| .
\end{aligned}
$$

Hence $\|x-y\|=\left\|x-T_{\lambda}^{n} z\right\|+\left\|T_{\lambda}^{n} z-y\right\|$, and consequently $T_{\lambda}^{n} z$ lies on the segment joining $x$ and $y$, by strict convexity. Since $T_{\lambda}^{n}$ is nonexpansive, $\left\|x-T_{\lambda}^{n} z\right\|=\left\|T_{\lambda}^{n} x-T_{\lambda}^{n} z\right\| \leqq\|x-z\|$, so that $T_{\lambda}^{n} z$ lies on the segment joining $x$ and $z$. Similarly, $T_{\lambda}^{n} z$ lies on the segment joining $z$ and $y$, which is possible only if $T_{\lambda}^{n} z=z$. That $F_{\lambda}^{n}$ is closed follows directly from the continuity of $T_{\lambda}$.

The mapping $U=\frac{1}{2}(I+T)$ is nonexpansive and has the same fixed points as $T$. The following theorem states conditions for the convergence of the sequence $\left\{U^{n} x\right\}$ to a fixed point of $U$ :

THEOREM A (EDELSTEIN [4]). Let $K$ be a closed convex subset of $a$ strictly convex Banach space $X, T: K \rightarrow K$ a nonexpansive mapping, and suppose $T(K)$ is contained in a compact subset $K_{1}$ of $K$. Then the sequence $\left\{U^{n} x\right\}$ converges to a fixed point of $T$ for all $x \in K$.

Suppose $\left\{T_{\lambda}\right\}_{\lambda \in \Lambda}$ is a commutative family each member of which satisfies the conditions of the theorem. If the set of fixed points of $T_{\lambda}$ is denoted by $F_{\lambda}$, then $F_{\lambda} \neq \varnothing$ for all $\lambda$ by the theorem. Under additional conditions on $\left\{F_{\lambda}\right\}$, the family $\left\{T_{\lambda}\right\}$ has a common fixed point.

THEOREM 1. Let $K$ be a closed convex subset of a strictly convex Banach space $X$ and $\left\{T_{\lambda}\right\}_{\lambda \in \Lambda}$ be a commutative family of mappings each member of which satisfies the conditions of the above theorem. Suppose for every $F_{\alpha}(\alpha \in \Lambda)$ having a positive diameter there exists $\lambda \in \Lambda$ such that $F_{\lambda}$ is a proper subset of $F_{\alpha}$. Then $\left\{T_{\lambda}\right\}$ has a common fixed point in $K$.

Proof. Let $x \in F_{\lambda}$. Then $\lim _{n \rightarrow \infty} U_{\mu}^{n} x=x_{0}$, where $U_{\mu} x_{0}=x_{0}$ by the theorem at sve. Since $x$ is a fixed point of $U_{\lambda}$ (and hence of $T_{\lambda}$ ), 
$\lim _{n \rightarrow \infty} U_{\lambda}\left(U_{\mu}^{n} x\right)=\lim _{n \rightarrow \infty} U_{\mu}^{n}\left(U_{\lambda} x\right)=x_{0}$, so that by the continuity of $U_{\lambda}, U_{\lambda} x_{0}=x_{0}$. Consequently, any two members of the family $\left\{T_{\lambda}\right\}_{\lambda \in \Lambda}$ have a fixed point in common.

Consider any decreasing chain of sets $\left\{F_{\alpha}\right\}$. Since the sets are closed by Proposition 1 and possess the finite intersection property, the family has a nonempty intersection by the compactness of the range of each $T_{\lambda}$.

Hence, by the Kuratowski-Zorn lemma, there exists a minimal element $F_{\lambda}$. If the diameter of $F_{\lambda}$ is positive, there exists $\alpha \in \Lambda$ such that $F_{\alpha}$ is a proper subset of $F_{\lambda}$, contradicting the minimality. Therefore $F_{\lambda}$ has exactly one element $x_{1}$, which is the unique fixed point of $T_{\lambda}$.

Since any two mappings have a fixed point in common, $x_{1}$ is a common fixed point of $\left\{T_{\lambda}\right\}_{\lambda \in \Lambda}$.

The following theorem gives conditions for the convergence of $\left\{T^{n} x\right\}$ to a fixed point of $T$ :

Theorem B (Browder And Petryshyn [2]). Let $C$ be a closed subset of a Banach space $X$. If the mapping $T: C \rightarrow C$ is nonexpansive,

$$
\lim _{n \rightarrow \infty}\left\|T^{n+1} x-T^{n} x\right\|=0
$$

for all $x \in C$, and the mapping $I-T$ maps closed bounded subsets of $C$ into bounded subsets of $X$ and if the set of fixed points of $T$ in $C$ is nonempty, then for any $x$ in $C$ the sequence $\left\{T^{n} x\right\}$ is convergent to a fixed point of $T$.

Using this result the proof of Theorem 1 carries over into

THEOREM 2. Let $C$ be a compact subset of a Banach space $X$. If the members of the family $\left\{T_{\lambda}\right\}_{\lambda \in \Lambda}$ of mappings satisfy the hypothesis of Theorem $\mathrm{B}$, and if for every $F_{\alpha}(\alpha \in \Lambda)$ having a positive diameter there exists $\lambda \in \Lambda$ such that $F_{\lambda}$ is a proper subset of $F_{\alpha}$, then $\left\{T_{\lambda}\right\}$ has a common fixed point in C.

Using the notion of a fixed point of order $n$, we get

THeOREM 3. Let $C$ be a closed subset of a Banach space, and $\left\{T_{\lambda}\right\}_{\lambda \in \Lambda} a$ commutative family of continuous functions from $C$ into itself. Suppose for every $x \in C,\left\{T_{\lambda}^{n} x\right\}$ converges to a fixed point of order $m$ of $T_{\lambda}$ for every $\lambda \in \Lambda$. Then $\left\{T_{\lambda}\right\}$ has a common fixed point of order $m$ in $C$.

Proof. Let $x \in F_{\lambda}^{m}$. Then $\lim _{n \rightarrow \infty} T_{\mu}^{n} x=x_{0}$, where $T_{\mu}^{m} x_{0}=x_{0}$, and $\lim _{n \rightarrow \infty} T_{\lambda}^{m}\left(T_{\mu}^{n} x\right)=\lim _{n \rightarrow \infty} T_{\mu}^{n}\left(T_{\lambda}^{m} x\right)=x_{0}$. Hence, by continuity, $T_{\lambda}^{m} x_{0}=x_{0}$, and $x_{0} \in F_{\lambda}^{m} \cap F_{\mu}^{m}$.

Suppose $\Lambda$ has been well-ordered and $\left\{\lambda_{1}, \lambda_{2}, \cdots, \lambda_{v}, \cdots, \lambda_{\alpha}, \cdots\right\}$ is a transfinite sequence from $\Lambda$. Suppose further that $F^{m}=F_{\lambda_{1}}^{m} \cap F_{\lambda_{2}}^{m} \cap \cdots \cap F_{\lambda_{\beta}}^{m}$ 
for all $\beta<\alpha$ is nonempty. Consider $T_{\lambda_{\alpha}}$ and $x \in F^{m}$; by hypothesis, $\left\{T_{\lambda_{\alpha}}^{n} x\right\}$ converges to a fixed point $x_{2}$ of order $m$ of $T_{\lambda_{\alpha}}$, i.e., $x_{2} \in F_{\lambda_{\alpha}}^{m}$. But $x_{2} \in F^{m}$ by the first part, so that $F^{m} \cap F_{\lambda_{\alpha}}^{m}$ is also nonempty.

THEOREM 4. Let $\left\{T_{\lambda}\right\}_{\lambda \in \Lambda}$ be a commutative family of operators from a subset $C$ of a Banach space into itself, and let $x_{0}$ be a common fixed point. Suppose for every $y \in C$, there exists $\lambda \in \Lambda$ such that $\lim _{n \rightarrow \infty} T_{\lambda .}^{n} y=x_{0}$. Let $T: C \rightarrow C$ be a continuous operator commuting with $\left\{T_{\lambda}\right\}$; then

(a) if $x$ is an rth order fixed point of $T$ (for some $x \in C$ ), $x_{0}$ is also an rth order fixed point of $T$;

(b) if $\lim _{n \rightarrow \infty} T_{\lambda}^{n} x=x_{0}$ for all $\lambda$ and some $x \in C, x_{0}$ is an ordinary fixed point of $T$.

Proof. (a) Consider $T^{n} x, n=1,2, \cdots$. By hypothesis,

$$
\lim _{m \rightarrow \infty} T_{\lambda_{n}}^{m}\left(T^{n} x\right)=x_{0}, \quad \lambda_{1}, \lambda_{2}, \cdots \in \Lambda,
$$

and by commutativity,

$$
\lim _{m \rightarrow \infty} T^{n}\left(T_{\lambda_{n}}^{m} x\right)=x_{0}, \quad \lambda_{1}, \lambda_{2}, \cdots \in \Lambda .
$$

Now if $x$ is an $r$ th order fixed point of $T, T^{r} x=x$ and $\lim _{m \rightarrow \infty} T_{\lambda_{r}}^{m}\left(T^{r} x\right)=x_{0}$ (i.e., for $n=r, \lim _{m \rightarrow \infty} T_{\lambda_{n}}^{m} x=x_{0}$ ). Hence by continuity $T^{r} x_{0}=x_{0}$.

(b) If $\lim _{n \rightarrow \infty} T_{\lambda}^{n} x=x_{0}$ for all $\lambda, T^{n} x_{0}=x_{0}$ for all $n$, and $x_{0}$ is an ordinary fixed point of $T$.

Note. If $C$ is a bounded closed set with "normal structure" (i.e., there exists a point in $C$ which is not diametral) of a reflexive and strictly convex Banach space, and if the mappings are nonexpansive, the assumption that $\left\{T_{\lambda}\right\}$ has a common fixed point follows and may be omitted (Browder [1]). Similarly, the assumption may be omitted if the mappings are nonexpansive and $C$ is compact and convex (De Marr [3]).

Returning to the question of computing the common fixed point, one can obtain a condition in terms of higher order fixed points.

THEOREM 5. Let $\left\{T_{\lambda}\right\}_{\lambda \in \Lambda}$ be a commutative family of operators on a subset $C$ of a Banach space into itself, and let $x_{0}$ be a common fixed point. Suppose that for every $x \in C$ there exists a $\lambda \in \Lambda$ such that $\lim _{m \rightarrow \infty} T_{\lambda}^{m} x=x_{0}$, and suppose further that $\left\{T_{\lambda}^{m} x\right\}$ converges for all $\lambda$. Let $T: C \rightarrow C$ be a continuous one-to-one operator commuting with the family $\left\{T_{\lambda}\right\}$ and such that $T^{r} x_{0}=x_{0}$ for some $r$. Then there exists $\alpha \in \Lambda$ such that $\lim _{m \rightarrow \infty} T_{\alpha}^{m} x=x_{0}$ for every $x \in C$.

Proof. Consider $T^{n} x, n=1,2, \cdots$. By hypothesis

$$
\lim _{m \rightarrow \infty} T_{\lambda_{n}}^{m}\left(T^{n} x\right)=x_{0}, \quad \lambda_{1}, \lambda_{2}, \cdots \in \Lambda,
$$


and

$$
\lim _{m \rightarrow \infty} T^{n}\left(T_{\lambda_{n}}^{m} x\right)=x_{0}, \quad \lambda_{1}, \lambda_{2}, \cdots \in \Lambda
$$

Since $\left\{T_{\lambda_{n}}^{m} x\right\}$ converges and $T$ is one-to-one, while $T^{r} x_{0}=x_{0}$ for some $r$, $\lim _{m \rightarrow \infty} T_{\lambda_{r}}^{m} x=x_{0}$ for all $x \in C$ and some $\lambda_{r} \in \Lambda$.

More interesting consequences are:

COROLlaRY 1. If $x_{0}$ is an ordinary fixed point of $T$, there exists a countably infinite subfamily $\left\{T_{\lambda_{n}}\right\}$ of $\left\{T_{\lambda}\right\}_{\lambda_{n \Lambda}}$ such that for every $x \in C$, $\lim _{m \rightarrow \infty} T_{\lambda_{n}}^{m} x=x_{0}, \lambda_{1}, \lambda_{2}, \cdots \in \Lambda$ (for then $T^{n} x_{0}=x_{0}$ for all $\left.n\right)$.

Corollary 2. If $T_{\lambda}$ is continuous and one-to-one for some $\lambda \in \Lambda$, the conclusion of Corollary 1 also follows.

3. Fixed points of $H H_{w}$-closed mappings. The following definition is due to Browder and Petryshyn [2]:

Definition 5. Let $H$ be a subset of a Banach space. A mapping $T: H \rightarrow H$ will be called $H_{w} H$-closed (demiclosed in the terminology of [2]) if its graph in $H \times H$ is closed in the Cartesian product topology induced in $H \times H$ by the weak topology in $H$ (denoted by $H_{w}$ ) and the strong topology in $H$.

In analogous fashion we shall define $H H_{w}$-closed:

Definition 6. Let $H$ be a subset of a Banach space. A mapping $T: H \rightarrow H$ will be called $H H_{w}$-closed if for any sequence $\left\{x_{n}\right\} \subset H$ which converges strongly to $x$ in $H$ the weak convergence of the sequence $\left\{T x_{n}\right\}$ to $y$ in $H$ implies that $T x=y$.

THEOREM 6. Let $H$ be a closed subset of a Banach space and $T$ a mapping from $H$ to $H$. Assume that $I-T$ is $H_{w^{-}}$-closed and $(I-T)^{-1}$ exists and is continuous from the weak topology in $R(I-T)$ to the strong topology in $H$. Assume further that there exists a sequence $\left\{x_{n}\right\} \subset H$ such that $\left\{(I-T) x_{n}\right\}$ is weakly convergent to 0 as $n \rightarrow \infty$. Then Thas a fixed point in $H$.

Proof. Let $\left\{x_{n}\right\}$ be the sequence for which $\left\{(I-T) x_{n}\right\}$ converges weakly to 0 . Since $(I-T)^{-1}$ is continuous from the weak topology in $R(I-T)$ to the strong topology in $H,\left\{x_{n}\right\}$ converges strongly to some $x_{0}$ as $n \rightarrow \infty$, where $x_{0} \in H$, since $H$ is closed. Because $I-T$ is $H H_{w}$-closed, $(I-T) x_{0}=0$, so that $T x_{0}=x_{0}$.

Another variation of this theorem is

THEOREM 7. Let $H$ be a closed subset of a Banach space and $T$ a mapping from $H$ to $H$. Assume that $I-T$ is $H H_{w}$-closed and $(I-T)^{-1}$ exists and is continuous from the weak topology in $R(I-T)$ to the strong 
topology in $H$. Finally, assume that for every $x \in H$ the sequence $\left\{T^{n} x-\right.$ $\left.T^{n+1} x\right\}$ is weakly convergent to 0 as $n \rightarrow \infty$. Then $T$ has a fixed point in $H$.

Proof. Consider the sequence $\left\{T^{n} x\right\}$. By hypothesis $\left\{\left(T^{n}-T^{n+1}\right) x\right\}=$ $\left\{(I-T) T^{n} x\right\}$ converges weakly to 0 as $n \rightarrow \infty$. Since $(I-T)^{-1}$ is continuous in the above sense and $I-T$ is $H H_{w}$-closed, $\left\{T^{n} x\right\}$ converges strongly to some $x_{0} \in H$, and $(I-T) x_{0}=0$.

Since $\left\{T^{n} x\right\}$ may be considered a sequence of successive approximations, we obtain from the proof of Theorem 7 the following

COROllary 3. For every $x \in H$ the sequence $\left\{T^{n} x\right\}$ of successive approximations converges to a fixed point of $H$.

THEOREM 8. Let $H$ be a compact subset of a Banach space, and let $\left\{T_{\alpha}\right\}_{\alpha \in \Lambda}$ be a commutative family of mappings from $H$ to $H$. For each $\alpha \in \Lambda$ assume that $I-T_{\alpha}$ is $H H_{w}$-closed and $\left(I-T_{\alpha}\right)^{-1}$ continuous from the weak topology in $R\left(I-T_{\alpha}\right)$ to the strong topology in $H$. Assume further that for every $x \in H$ and $\alpha \in \Lambda$ the sequence $\left\{\left(T_{\alpha}^{n}-T_{\alpha}^{n+1}\right) x\right\}$ converges weakly to 0 as $n \rightarrow \infty$. Then $\left\{T_{\alpha}\right\}_{\alpha \in \Lambda}$ has a common fixed point in $H$.

Proof. Let $K_{\alpha}$ be the set of fixed points of $T_{\alpha}$. Then $K_{\alpha} \neq \varnothing$ for every $\alpha \in \Lambda$ by Theorem 7 .

Let $\left\{x_{n}\right\}$ be a sequence in $K_{\alpha}$ converging strongly to $x$ as $n \rightarrow \infty$. Then $\left\{T_{\alpha} x_{n}\right\}$ converges strongly to $x$ (and hence weakly), so that $\left\{\left(I-T_{\alpha}\right) x_{n}\right\}$ converges weakly to 0 as $n \rightarrow \infty$. Since $I-T_{\alpha}$ is $H H_{w}$-closed, $\left(I-T_{\alpha}\right) x=0$, i.e., $T_{\alpha} x=x$. Consequently, $K_{\alpha}$ is closed for every $\alpha \in \Lambda$.

Since $H$ is compact, it is sufficient to show that $\left\{K_{\alpha}\right\}_{\alpha \in \Lambda}$ has the finite intersection property.

Toward this end we first observe that for any $\alpha \in \Lambda$ and $x \in K_{\alpha}$

$$
T_{\alpha}\left(T_{\lambda} x\right)=T_{\lambda}\left(T_{\alpha} x\right)=T_{\lambda} x
$$

by commutativity. Hence for any $\alpha \in \Lambda, T_{\lambda}$ maps $K_{\alpha}$ into itself.

Proceeding by induction, we choose any sequence $\alpha_{1}, \alpha_{2}, \cdots, \alpha_{n}$ from $\Lambda$ with $K=K_{\alpha_{1}} \cap K_{\alpha_{2}} \cap \cdots \cap K_{\alpha_{n-1}}$ assumed nonempty. Recalling that $T_{\alpha_{n}}$ maps $K_{\alpha_{i}}(i=1,2, \cdots, n-1)$ into itself, we may consider $T_{\alpha_{n}}$ to be a mapping from $K$ to $K$. Since $K$ is closed, $K_{\alpha_{n}} \neq \varnothing$ by Theorem 7, so that $K \cap K_{\alpha_{n}}$ is also nonempty.

\section{REFERENCES}

1. F. E. Browder, Nonexpansive nonlinear operators in a Banach space, Proc. Nat. Acad. Sci. U.S.A. 54 (1965), 1041-1044. MR 32 \#4574.

2. F. E. Browder and W. V. Petryshyn, The solution by iteration of nonlinear functional equations in Banach spaces, Bull. Amer. Math. Soc. 72 (1966), 571-575. MR 32 \#8155b. 
3. R. De Marr, Common fixed points for commuting contraction mappings, Pacific J. Math. 13 (1963), 1139-1141. MR 28 \#2446.

4. M. Edelstein, A remark on a theorem of M. A. Krasnoselski, Amer. Math. Monthly 73 (1966), 509-510. MR 33 \#3072.

5. W. A. Kirk, A fixed point theorem for mappings which do not increase distance, Amer. Math. Monthly 72 (1965), 1004-1006. MR 32 \#6436.

Department of Mathematics, Milwaukee School of Engineering, Milwaukee, WISCONSIN 53202 